\title{
ESTUDIO COMPARATIVO DE MORFOLOGÍA Y PROPIEDADES FÍSICO- MECÁNICAS ENTRE EL POLIPROPILENO Y COMPUESTOS DE POLI- PROPILENO REFORZADO CON SÍLICE DE DISTINTA PROCEDENCIA Y CÁSCARA DE ARROZ
}

\author{
O. Murillo Tapia ${ }^{1-3}$ P. Toro ${ }^{2}$, Y. Pedram², R. Quijada ${ }^{2}$, L. Aquice ${ }^{3}$
}

\section{RESUMEN}

Se estudió la influencia de sílice de distinta procedencia y de cáscara de arroz incorporadas dentro de una matriz de polipropileno, evaluando el comportamiento físico-mecánico y morfológico de estos materiales compuestos, también evaluamos el efecto de compatibilizantes en la interfase. Establecemos que a mayor contenido de sílice, aumenta el módulo de Young, el porcentaje de absorción agua, la densidad aparente y disminuye la elongación a la ruptura, la resistencia al impacto y el índice de fluidez de los compuestos. El efecto del compativilizante VTES es mas eficiente en la interfase PP/sílice respecto al compativilizante MMI. Así mismo, establecemos que a mayor contenido de cáscara de arroz disminuye el módulo de Young, pero al utilizar compatibilizante MMI el módulo de Young y la resistencia a la tracción aumentan, consecuentemente la densidad aparente y el porcentaje de absorción de agua tienden a aumentar pero la resistencia al impacto y el índice de fluidez disminuyen, pero sus valores son mas representativos comparados a los compuestos PP/sílice.

Todos los resultados están en función al incremento de sílice o cáscara de arroz en la matriz de polipropileno. Respecto al análisis térmico destacamos que con $50 \%$ de cáscara de arroz dentro de la matriz de polipropileno no existe variación de su temperatura de transición vítrea y temperatura de fusión.

\section{INTRODUCCIÓN}

El Polipropileno (PP) es un polímero termoplástico que presenta mayor crecimiento de consumo en la última década. La optimización de sus propiedades para ampliar sus aplicaciones requiere mejorar sus propiedades mecánicas y térmicas. Esto conduce a la necesidad de desarrollar materiales compuestos en base de PP y materiales de refuerzo. En este contexto el uso de sílice como material de refuerzo y polipropilenos funcionalizados con monómeros polares como compatibilizantes es una vía para lograr este objetivo.

Sílice obtenida de la cáscara de arroz se ha utilizado como aditivo para mejorar las propiedades mecánicas en blendas PP/EPDM ${ }^{1}$, también ha sido el caso en mezclas de PP/caucho natural junto a un compatibilizante, 3aminopropil trietoxi silano, y polipropileno funcionalizado con ácido acríli$\mathrm{co}^{2}$. Este mismo efecto se ha observado en compuestos de PP/cáscara de arroz ${ }^{3}$, así

como la influencia de agentes de acoplamiento como silanos organofuncionales en compuestos de PP reforzados con fibra de vidrio ${ }^{4}$. En este trabajo se estudio la influencia de sílice de distinta procedencia y de cáscara de arroz en el comportamiento mecánico (módulo de tracción y deformación en la ruptura, resistencia al impacto), físico (absorción de agua, densidad aparente, índice de fluidez, análisis térmico diferencial) y morfológico de materiales compuestos con matriz de PP y/o PP- funcionalizado con monometil itaconato 0 vinil trietoxisilano.

\section{MATERIAL Y MÉTODOS}

- Polipropileno Heterofásico (PP) (PCC0610), Petroquim-Chile; $9.5 \%$ de etileno; $\mathrm{MFI}=6.2$

- Compatibilizantes: i) PP injertado con $1.5 \%$ de monometilitaconato en fase fundida (MMI), y ii) PP injertado con $2 \%$ de vinil-trietoxi silano en fase fundida (VTES).

- Silice: i)Comercial Crosfield Group Warrintong (EP10X y EP17G), ii) Sintética a partir de Metasilicatos (VSSiM) sintetizada en laboratorio de Investigación de la Universidad de Chile, y iii) Natural a partir de la calcinación $\left(700^{\circ} \mathrm{C} / 3 \mathrm{hrs}\right)$ de cáscara de arroz (CASi).

- Cáscara de arroz (CA): tamizada (malla 10-14), lavada y secada.

Las sílices y la cáscara de arroz se caracterizaron por análisis granulométrico (Malvern Master Sizer), superficie específica (BET) y morfológico (SEM). Los compositos de matriz PP se obtuvieron en un mezclador
Brabender-Plasticorder $\left(190^{\circ} \mathrm{C}, \quad 75\right.$ $\mathrm{rpm} ; 5 \mathrm{~min}$.) en presencia de antioxidante Irganox1010/Irgafos168 (2/1) y ambiente de nitrógeno para evitar la oxidación del compuesto durante el proceso de mezcla. La mezcla resultante fue peletizada y moldeadas a $190^{\circ} \mathrm{C}$ para obtener las placas y las probetas para su caracterización.

La composición de los compositos estudiados son: PP/sílice y PP/CA:

- PP con $10 \%$ y $30 \%$ en peso de sílice (EP10X, EP17G, VSSiM, CASi)

- PP con $10 \%, 30 \%$ y $50 \%$ en peso de CA

- PP/PP-funcionalizado/sílice y PP/ PP-funcionalizado/CA:

- PP reforzado con $10 \%$ en peso de sílice EP10X y $5 \%$ en peso de compatibilizante (MMI y VTES), y

- PP con $10 \%, 30 \%$ y $50 \%$ en peso de CA, y $5 \%$ en peso de compatibilizante (MMI)

Los ensayos determinados fueron: resistencia a la tracción (módulo de Young y alargamiento a la ruptura ; ASTM D-638), resistencia al impacto (ISO-179), absorción de agua (ISO62), densidad aparente (DIN - 53 479) , índice de fluidez (ASTM D-1238), calorimetría diferencial de barrido (ASTM D-3417) y análisis morfológico (SEM).

\footnotetext{
${ }^{1}$ SGS del Perú S.A.C.-Lima, Perú E-mail: ofmurillotapia@yahoo.com, ${ }^{2}$ Fac. Cs. Fís. y Mat.-Depto. Ing. Quím.-Univ. de Chile-Santiago-Chile, Ing. Mat.Fac. Ing. Proc.-UNSA-Arequipa, Perú
} 


\section{RESULTADOS Y DISCUSIÓN}

En tabla 1 son presentados los resultados de módulo de Young y la elongación a la ruptura para los compositos PP/sílice preparados. De estos resultados se puede establecer el efecto reforzante presentado por las sílices de distinta procedencia al aumentar la rigidez de los compositos, debido al mayor valor del módulo de Young respecto al PP. Además, se ha observado que a medida que aumenta el contenido de las sílices, de $10 \%$ a $30 \%$ en peso, se produce un incremento del módulo hasta un $20 \%$ en los compositos de PP/sílice. Sin embargo, en todos los casos el aumento de la rigidez va acompañado con una disminución apreciable de la elongación a la ruptura respecto al PP.

En relación al efecto de los agentes compatibilizantes MMI y VTES en los compositos PP/sílice EP10X que se muestra en la tabla 2 , se ha determinado, un aumento apreciable del módulo de Young al utilizar el compatibilizante VTES con un $5 \%$ en peso. Por otro lado, al utilizar el compatibilizante MMI no se a logrado una mejor adhesión de la sílice EP10X con el PP dado el despreciable aumento del módulo observado, respecto al PP/ sílice o PP

Sin embargo, en el caso de los compositos PP/CA, el compatibilizante MMI es un agente de acoplamiento apropiado para lograr un mejoramiento de la adhesión de la CA con el PP debido al aumento considerable observado en la rigidez de los compositos PP/CA/MMI respecto al PP/CA para contenidos de CA desde $10 \%$ a $50 \%$ en peso (tabla 3 ).

La diferencia observada del efecto de los agentes compatibilizantes (MMI y

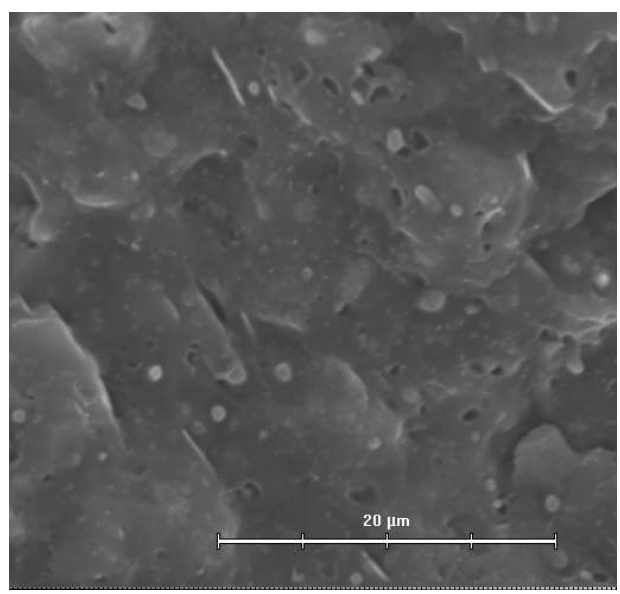

PP / EP10X 90/10:3300X
VTES), para los reforzantes como es el caso de la sílice EP10X y la CA en los compositos PP/sílice o PP/CA puede estar asociada a la naturaleza diferente de los reforzantes.

El grado de adhesión y el mejoramiento de la distribución de los agentes reforzantes, como son las sílices

\begin{tabular}{|c|c|c|}
\hline \multirow{2}{*}{ Compuestos } & Módulo de Young & Elongación a la ruptura \\
\cline { 2 - 3 } & $\mathbf{( M p a )}$ & $\mathbf{( \% )}$ \\
\hline PP & 890 & 74 \\
\hline PP-EP10X(10\%) & 979 & 16 \\
\hline PP-EP17G(10\%) & 1019 & 20 \\
\hline PP-VSSiM(10\%) & 1007 & 22 \\
\hline PP-CASi(10\%) & 955 & 26 \\
\hline PP-EP10X(30\%) & 1074 & 5 \\
\hline PP-EP17G(30\%) & 1069 & 6 \\
\hline PP-VSSiM(30\%) & 1059 & 9 \\
\hline PP-CASi(30\%) & 1060 & 6 \\
\hline
\end{tabular}

Tabla 1: Módulo de Young y Elongación a la ruptura de Compuestos PP/Sílice (EP10X, EP17G, VSSiM y CASi).

\begin{tabular}{|c|c|c|}
\hline \multirow{2}{*}{ Compuestos } & Módulo de Young & Elongación a la ruptura \\
\cline { 2 - 3 } & $\mathbf{( M P a )}$ & $\mathbf{( \% )}$ \\
\hline PP & 890 & 74 \\
\hline PP-EP10X(10\%) & 979 & 16 \\
\hline PP-MMI(5\%)-EP10X(10\%) & 905 & 16 \\
\hline PP-VTES(5\%)-EP10X(10\%) & 1021 & 14 \\
\hline
\end{tabular}

Tabla 2: Módulo de Young y elongación a la ruptura de Compuestos PP/Sílice EP10X con $10 \%$ en peso de sílice y $5 \%$ en peso de compatibilizante (MMI y VTES).

\begin{tabular}{|c|c|c|}
\hline \multirow{2}{*}{ Compuestos } & Módulo de Young & Elongación a la ruptura \\
\cline { 2 - 3 } & (Mpa) & $(\%)$ \\
\hline PP & 890 & 74 \\
\hline PP-CA(10\%) & 829 & 6 \\
\hline PP-CA(30\%) & 774 & 3 \\
\hline PP-CA(50\%) & 715 & 3 \\
\hline PP-MMI(5\%)-CA(10\%) & 965 & 5 \\
\hline PP-MMI(5\%)-CA(30\%) & 1105 & 4 \\
\hline PP-MMI(5\%)-CA(50\%) & 1181 & 3 \\
\hline
\end{tabular}

Tabla 3: Módulo de Young y elongación a la ruptura de Compuestos PP/CA con 10, 30 y $50 \%$ en peso de CA y $5 \%$ en peso de compatibilizante (MMI).

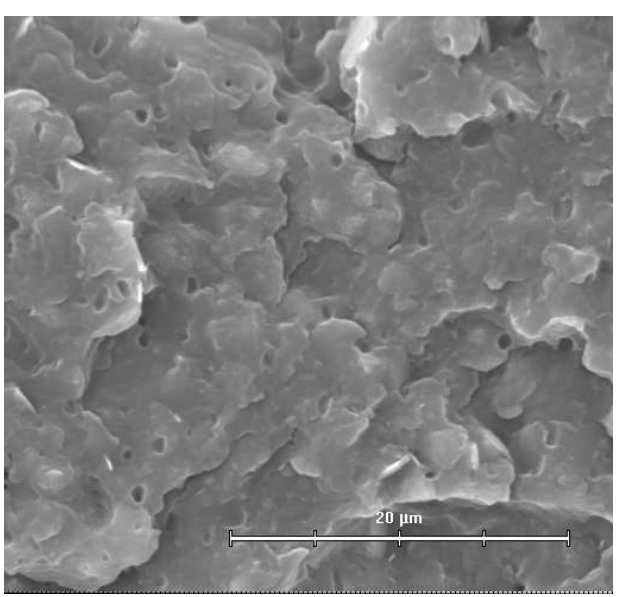

PP/ VTES/EP10X 85/5/10: 3300X

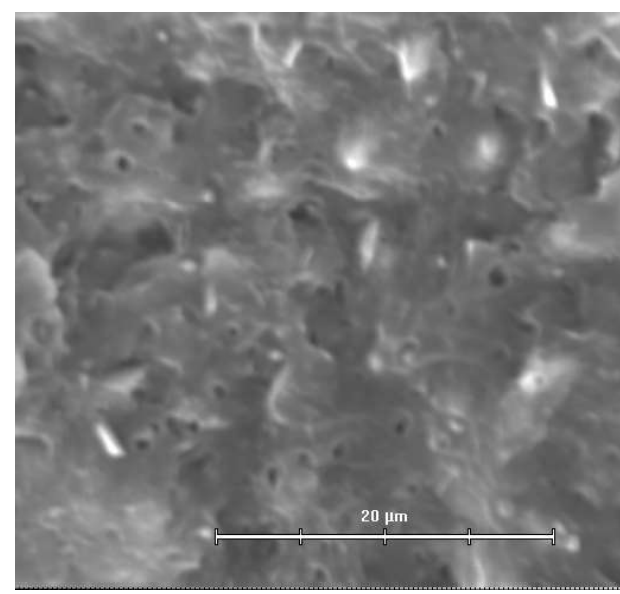

$\mathrm{PP} / \mathrm{MSi} \sim 90 / 10: 3300 \mathrm{X}$ 

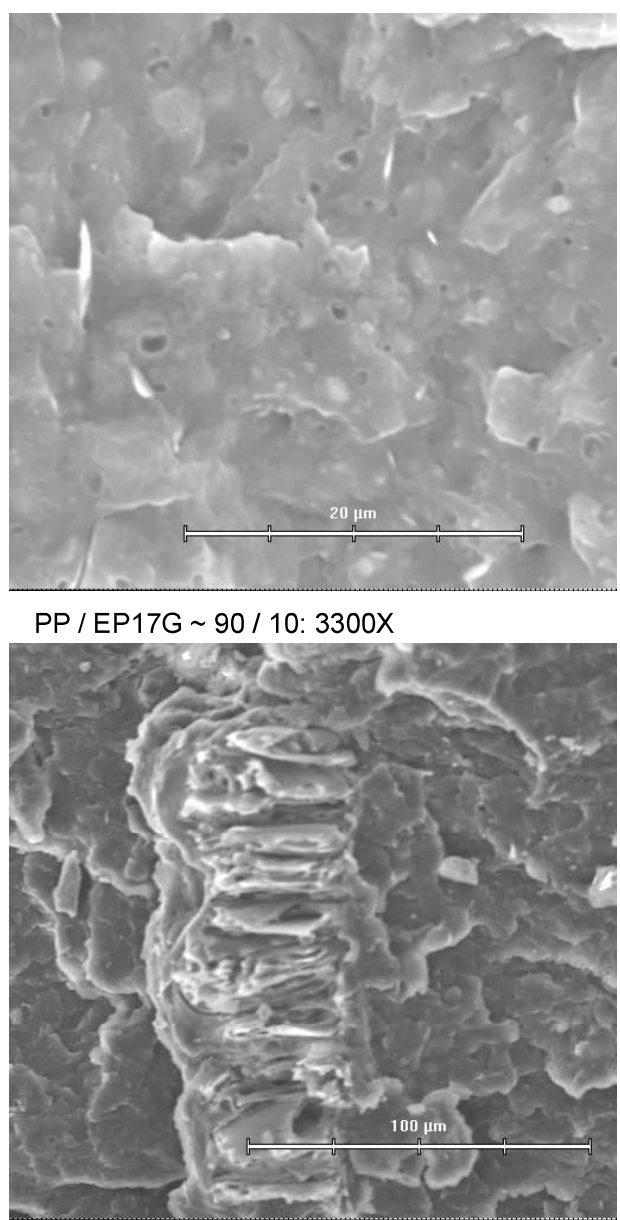

$\mathrm{PP} / \mathrm{MMI} / \mathrm{CA} \sim 85$ / 5 / 10: 670X

Por último, una caracterización adicional de los compositos elaborados ha sido la determinación de la resistencia al impacto, absorción de agua densidad aparente, índice de fluidez, análisis diferencial térmico, como se muestra en la tabla 4 . De estos resultados se puede observar una tendencia a aumentar la densidad aparente, absorción de agua y una tendencia a disminuir la resistencia al impacto, índice de fluidez, estos resultados están en función del contenido y tipo de agente reforzante. En este caso también se ha observado la diferencia de naturaleza entre la CA y las sílices comerciales como agentes reforzantes, debido al distinto grado de asociación de agua que le confieren finalmente al composito. Así mismo podemos establecer que según los diagramas obtenidos del DSC, la temperatura de cristalización vítrea, temperatura de fusión y entalpía permanece inalterable respecto al PP.

\section{AGRADECIMIENTO}

A Dios y a los Doctores Raúl Quijada, Patricio Toro, Yazdani Pedram Docentes de la Universidad de Chile y a la Comisión Organizadora del XI $\mathrm{ECl}-2004 \mathrm{v}$.

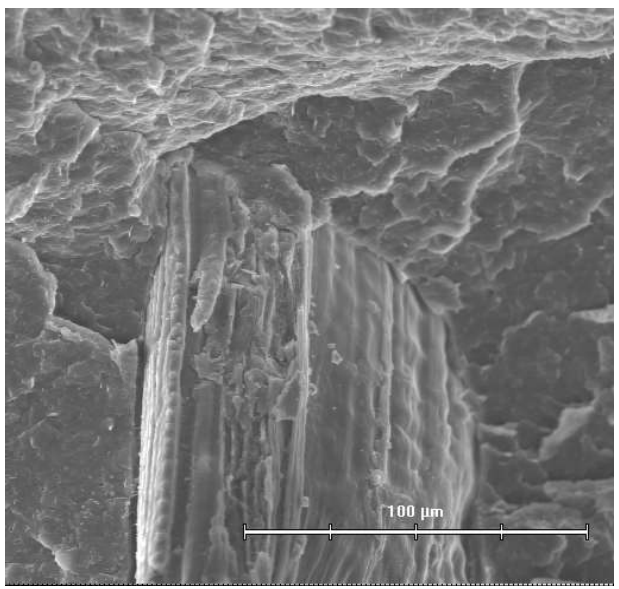

$\mathrm{PP} / \mathrm{CA} \sim 90 / 10: 670 \mathrm{X}$

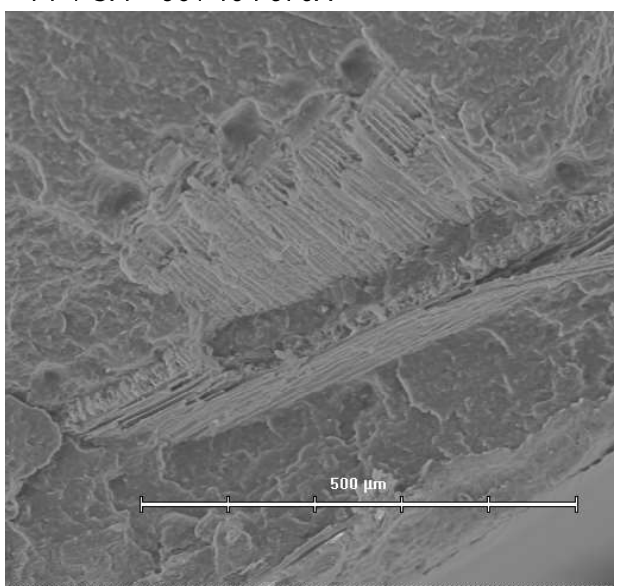

$\mathrm{PP} / \mathrm{MMI} / \mathrm{CA} \sim 65$ / 5 / 30: 170X

$\mathrm{PP} / \mathrm{CAC} \sim 90 / 10: 3300 \mathrm{X}$

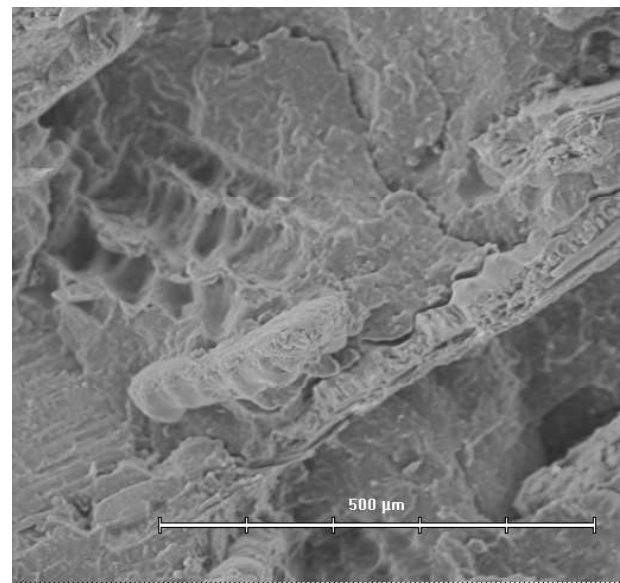

$\mathrm{PP} / \mathrm{CA} \sim 70 / 30: 170 \mathrm{X}$

Figura 1: SEM de los compuestos Elaborados

\begin{tabular}{|c|c|c|c|c|}
\hline \multirow{2}{*}{ Compuestos } & $\begin{array}{c}\text { Resistencia } \\
\text { Impacto }\end{array}$ & $\begin{array}{c}\text { Índice de } \\
\text { Fluidez }\end{array}$ & Densidad & $\begin{array}{c}\text { Absorción } \\
\text { de Agua }\end{array}$ \\
\cline { 2 - 5 } & $\mathbf{J} / \mathbf{m}$ & $\mathbf{g} / \mathbf{1 0 m i n}$ & $\mathbf{g} / \mathbf{c m}^{\mathbf{3}}$ & $\mathbf{( \% )}$ \\
\hline $\mathrm{PP}$ & 338 & 6.53 & 0.81 & 0.1 \\
\hline PP-EP10X(10\%) & 252 & 2.45 & 0.95 & 0.6 \\
\hline PP-EP17G(10\%) & 257 & 2.77 & 0.90 & 0.8 \\
\hline PP-VSSiM(10\%) & 232 & 2.82 & 0.91 & 0.7 \\
\hline PP-CASi(10\%) & 262 & 3.01 & 0.90 & 0.2 \\
\hline PP-EP10X(30\%) & 246 & 0.00 & 1.06 & 4.8 \\
\hline PP-EP17G(30\%) & 249 & 0.00 & 1.25 & 5.1 \\
\hline PP-VSSiM(30\%) & 221 & 0.00 & 1.16 & 3.3 \\
\hline PP-CASi(30\%) & 254 & 0.00 & 1.05 & 1.2 \\
\hline PP-CA(10\%) & 222 & 6.50 & 0.90 & 0.8 \\
\hline PP-MMI(5\%)-CA(10\%) & 249 & 6.60 & 0.85 & 0.5 \\
\hline PP-CA(30\%) & 210 & 4.40 & 1.00 & 3.0 \\
\hline PP-MMI(5\%)-CA(30\%) & 267 & 4.50 & 0.96 & 1.9 \\
\hline PP-CA(50\%) & 196 & 3.30 & 1.27 & 4.5 \\
\hline PP-MMI(5\%)-CA(50\%) & 293 & 3.40 & 1.20 & 3.8 \\
\hline
\end{tabular}

Tabla 4: Resistencia al Impacto, Índice de Fluidez, Densidad Aparente y Absorción de Agua de Compuestos PP/Sílice (10\% y $30 \%$ en peso); y PP/CA (10\%, $30 \%$ y $50 \%$ en peso) con y sin compatibilizante MMI ( $5 \%$ en peso)

\section{REFERENCIAS BIBLIÓGRÁFICAS}

1. S. Siriwardena, H. Ismail, and U.S. Ishiaku, Polym. - Plast. Technol. Eng. 40(4),(2001)519.

2. Ismail, H., Mega, L., Polym. - Plast. Technol. Eng., 40(4)(2001)463.

3. Z. A. Mohd. Ishak, B.N. Yow, B.L. Ng, H.P.S.A., Khalil, H.D.Rozman, J. Of Applied Polymer Science, 81(3),(2001),742.

4. D. Bikiaris, P. Matzinos, A. Larena, V. Flaris, C. Panayiotou, J. Of Applied Polymer Science, 81(3), (2001), 701. 